\title{
Growth of three-dimensional silver fractal electrodeposits under damped free convection
}

\author{
P. Carro, S. L. Marchiano, A. Hernández Creus, S. González, R. C. Salvarezza, and A. J. Arvia \\ Departamento de Química Física, Facultad de Química, Universidad de La Laguna, Tenerife, Spain \\ and Instituto de Investigaciones Fisicoquímicas Téricas y Aplicadas (INIFTA), Universidad Nacional de La Plata, \\ La Plata, Argentina \\ (Received 20 January 1993)
}

\begin{abstract}
Quasispherical three-dimensional Ag electrodeposits grown at constant potential and damped free convection exhibit a quasi-isotropic radial growth up to a critical radius $r_{c} \cong 0.3 \mathrm{~cm}$ and a mass fractal dimension $D_{M}=2.5$. For $r \gg r_{c}, D_{M} \rightarrow 3$. The product $v_{r} \eta^{\prime}$ is nearly constant, $v_{r}$ being the radial growth velocity, and $\eta^{\prime}$ the apparent medium viscosity. It appears that $v_{r}$ is determined by the Laplacian field operating between cathode and anode.
\end{abstract}

PACS number(s): 68.70. $+\mathrm{w}$, 68.55.Jk, 82.20.Wt

The formation of a new solid phase through electrodeposition of material on conducting substrates leads to dense radial, needlelike, and branched aggregate structures, depending on the experimental conditions [1]. Since the pioneering works on the matter appeared $[2,3]$, there has been increasing interest in exploring the fractal nature of rough metal electrodeposits. Commonly, branched metal aggregates are grown using quasi-twodimensional (2D) electrochemical cells leading to quasi2D patterns $[1,3]$ which evolve from diffusion-limited aggregation (DLA) patterns with a fractal dimension $D_{M} \cong 1.7$ [4] to dense radial patterns with $D_{M} \cong 2$ [5].

Despite the relevance of 3D metal electrodeposits, the fractal characterization of these objects is seldom found in the literature. It was shown [2] that $3 \mathrm{D} \mathrm{Cu}$ electrodeposits in the micrometer size range behave as selfsimilar fractals with $D_{M}=2.43$, as predicted by DLA 3D models [4-7]. In contrast, very little is known about 3D objects with larger sizes than the corresponding diffusion layer thickness, and conclusions derived from 2D objects [8-10] were taken for granted for 3D systems $[11,12]$. The main problem in dealing with $3 \mathrm{D}$ electrodeposit growth in aqueous solutions is the interference of free convection, which tends to produce anisotropic growth patterns [13-16].

This Rapid Communication describes aspects related to constant-potential damped-free-convection grown 3D Ag electrodeposits with a final size on the order of $1 \mathrm{~cm}$. Results demonstrate the role played by the decrease of free convection in favoring the growth of isotropic electrodeposits with $D_{M}$ values compatible with the predictions of models in Laplacian fields [4]. A change of the deposit structure from DLA-like to a dense radial pattern can also be observed at advanced growth stages.

Runs were made using a Pt spherical cathode $(0.076$ $\mathrm{cm}$ average diameter, sphericity deviation less than $10 \%$ ) placed at the symmetry axis of a $3 \mathrm{D}$ cylindrical cell. The counterelectrode was a Ag spiral around the working electrode to approach a homogeneous primary current distribution. A saturated calomel electrode (SCE) was used as a reference.

The base solution was $0.50 M \quad \mathrm{Na}_{2} \mathrm{SO}_{4}+0.010 M$ $\mathrm{H}_{2} \mathrm{SO}_{4}+0.005 \mathrm{M} \mathrm{Ag}_{2} \mathrm{SO}_{4}$, and agarose (0.033-0.5 g/100 $\mathrm{ml}$ range) was added to cover both sol and gel ranges.
Sols behave as pseudoplastic fluids, with $\eta^{\prime}$ the apparent viscosity coefficient reading at $450-\mathrm{s}^{-1}$ shear rate, in the 1.61-2.18-P range.

$\mathrm{Ag}$ electrodeposits were made at constant potential $E_{d}=-0.200 \mathrm{~V}$ (vs SCE), i.e., $-0.580 \mathrm{~V}$ apart from the
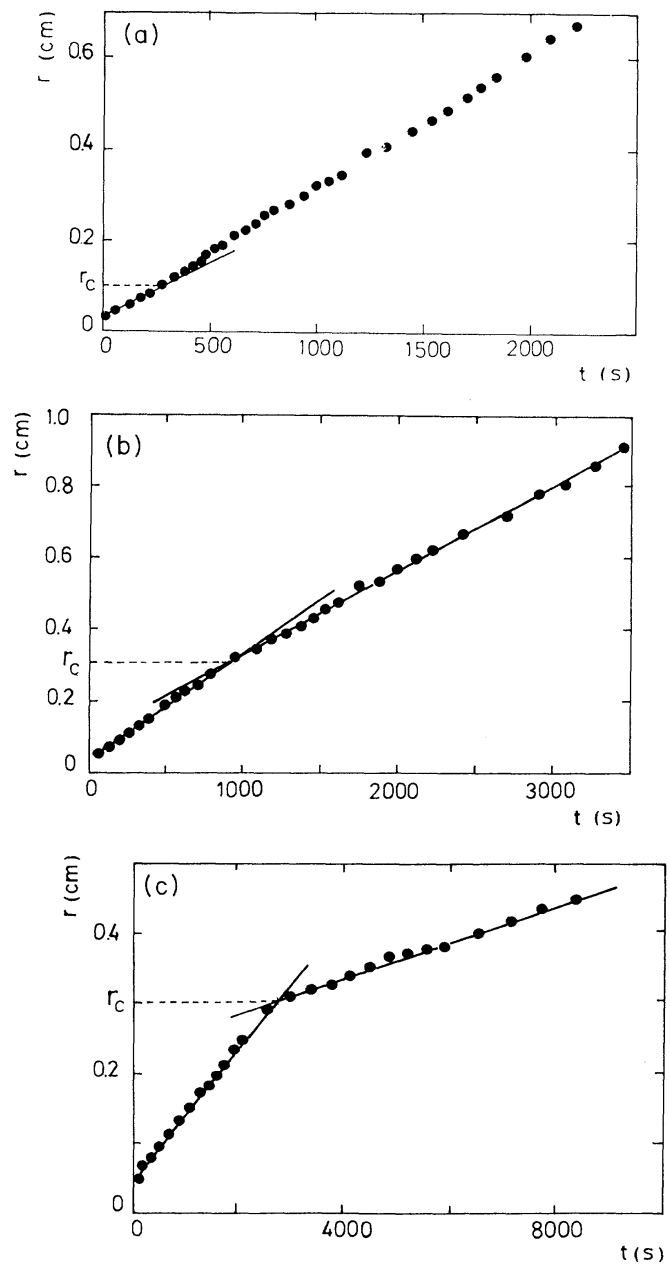

FIG. 1. Plots of $r$ vs time for silver electrodeposits. $E_{d}=-0.200 \mathrm{~V}, \quad 0.50 M \quad \mathrm{Na}_{2} \mathrm{SO}_{4}+0.010 M \quad \mathrm{H}_{2} \mathrm{SO}_{4}+0.005 M$ $\mathrm{Ag}_{2} \mathrm{SO}_{4}+x \mathrm{~g}$ agarose $/ 100 \mathrm{ml}, 25^{\circ} \mathrm{C}$. (a) $x=0$; (b) $x=0.07$; (c) $x=0.5$. 
equilibrium potential of the $\mathrm{Ag} / \mathrm{Ag}^{+}(\mathrm{aq})$ reversible electrode [17]. Current ( $I$ vs $t$ ) and the charge ( $Q$ vs $t$ ) transients were run with a Princeton Applied Research equipment. Runs were performed at $25^{\circ} \mathrm{C}$ under argon. A detailed description of the experimental procedure is given elsewhere [16-18].

In all solutions, the radius of gyration $(r)$ vs time $(t)$ plots display two different regions with a crossover defined at $\left(r_{c}, t_{c}\right)$, where $r_{c}$ is a critical radius involving a change in either the object shape and/or density, and $t_{c}$ is the time required to reach $r_{c}$.

In agarose-free solution the $r$ vs $t$ plot shows a linear portion (constant $v_{r}$ ) followed by another $r$ vs $t$ relationship [Fig. 1(a)] involving an increase in $v_{r}$ [16]. For $r<r_{c}$ a quasi-isotropic object grows, whereas for $r>r_{c}$ a pinecone-shaped object develops [Fig. 2(a)], although the object density remains constant with growth.

The $r$ vs $t$ plots of objects grown in sols [Fig. 1(b)] exhibit a first linear portion for $r<r_{c}$ with a $v_{r}$ value greater than that resulting for $r>r_{c}$. The value of $r_{c}$ determines the size of the object related to the quasi-

(a)

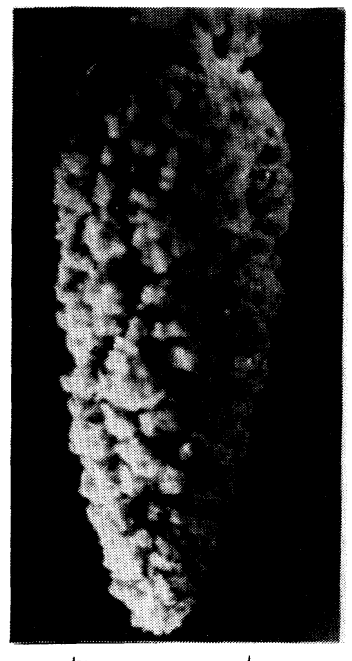

$0.35 \mathrm{~cm}$

(c)

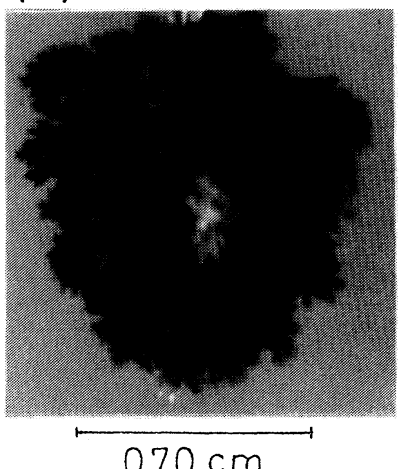

(d)

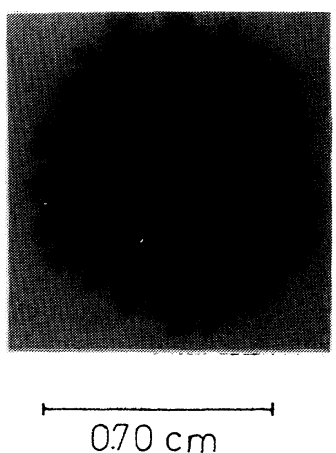

FIG. 2. Photographs of silver dendritic deposits. $E_{d}=-0.200 \quad \mathrm{~V}, \quad 0.50 M \quad \mathrm{Na}_{2} \mathrm{SO}_{4}+0.010 M \quad \mathrm{H}_{2} \mathrm{SO}_{4}+0.005 M$ $\mathrm{Ag}_{2} \mathrm{SO}_{4}+x \mathrm{~g}$ agarose $/ 100 \mathrm{ml}, Q=1 C, 25^{\circ} \mathrm{C}$. (a) $x=0$; (b) $x=0.033$; (c) $x=0.070$; (d) $x=0.5$.
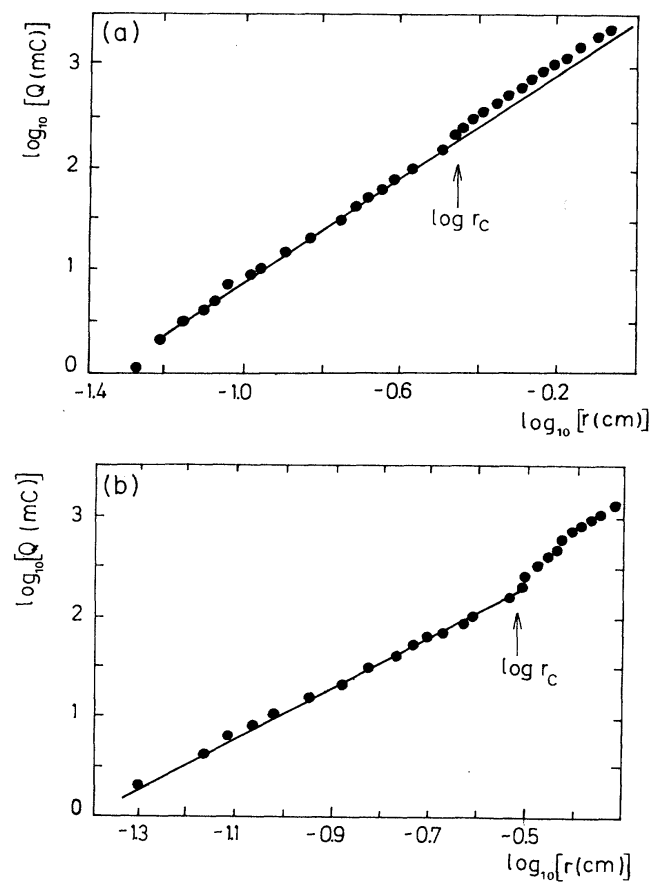

FIG. 3. $\log _{10} Q$ vs $\log _{10} r$ plots for silver electrodeposits, $E_{d}=-0.200 \mathrm{~V}, \quad 0.50 M \quad \mathrm{Na}_{2} \mathrm{SO}_{4}+0.010 M \quad \mathrm{H}_{2} \mathrm{SO}_{4}+0.005 M$ $\mathrm{Ag}_{2} \mathrm{SO}_{4}+x \mathrm{~g}$ agarose $/ 100 \mathrm{ml}, 25^{\circ} \mathrm{C}$. (a) $x=0.07$; (b) $x=0.5$.

isotropic to anisotropic growth transition.

As the agarose concentration is increased, 3D Ag branched aggregates grown up to $Q=1 \mathrm{C}$ [Figs. 2(a)-2(d)] change their contour from an anisotropic (conelike) to a quasi-isotropic (spherical) one. Simultaneously, the isotropic growth range extends to greater values of $Q$ due to free convection damping. Besides, for $r<r_{c}, v_{r}$ decreases with agarose concentration.

The $r$ vs $t$ plots for objects grown in the gel [Fig. 1(c)] are similar to those already described for sols, although in gel $v_{r}$ is smaller than in sols, and its change at $r_{c}$ takes place without an appreciable change in the object shape.

Ag electrodeposits grown in agarose-free solution show $D_{M}=3.0 \pm 0.03$ (Euclidean volume), and a surface fractal dimension, $D_{s}=2.5 \pm 0.05$ [16]. The value of $D_{M}$ of quasi-spherical $\mathrm{Ag}$ electrodeposits $\left(r<r_{c}\right)$ grown in agarose-containing media was determined from

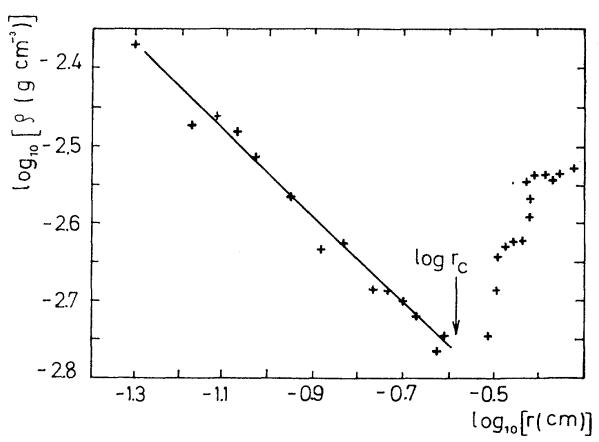

FIG. 4. $\log _{10} \rho$ vs $\log _{10} r$ plot for silver electrodeposits, $E_{d}=-0.200 \mathrm{~V}, \quad 0.50 M \quad \mathrm{Na}_{2} \mathrm{SO}_{4}+0.010 M \quad \mathrm{H}_{2} \mathrm{SO}_{4}+0.005 M$ $\mathrm{Ag}_{2} \mathrm{SO}_{4}+0.5 \mathrm{~g}$ agarose $/ 100 \mathrm{ml}, 25^{\circ} \mathrm{C}$. 
TABLE I. Parameters related to the growth of the macroscopic object, $0.50 \mathrm{M} \mathrm{Na} \mathrm{Na}_{4}+0.010 \mathrm{M}$ $\mathrm{H}_{2} \mathrm{SO}_{4}+0.005 \mathrm{M} \mathrm{Ag}_{2} \mathrm{SO}_{4}+x \mathrm{~g}$ agarose $/ 100 \mathrm{ml}$ solutions.

\begin{tabular}{|c|c|c|c|c|c|c|}
\hline $\begin{array}{c}x \\
(\mathrm{~g} / 100 \mathrm{ml}) \\
\end{array}$ & $\begin{array}{c}\eta^{\prime} \\
\left(10^{2} \mathrm{P}\right)\end{array}$ & $\begin{array}{c}v_{r} \\
\left(10^{4} \mathrm{~cm} \mathrm{~s}^{-1}\right)\end{array}$ & $\begin{array}{c}\eta^{\prime} v_{r} \\
\left(10^{6} \mathrm{P} \mathrm{cm} \mathrm{s}^{-1}\right)\end{array}$ & $\begin{array}{c}\delta \\
\left(D_{i} / v_{r}\right) \\
(\mathrm{cm}) \\
\end{array}$ & $\begin{array}{c}r_{c} \\
(\mathrm{~cm}) \\
\end{array}$ & $\begin{array}{c}D_{M} \\
\left(r<r_{c}\right) \\
\end{array}$ \\
\hline 0 (blank) & 1.130 & 2.80 & 3.16 & 0.038 & 0.10 & $3.00^{\mathrm{a}}$ \\
\hline 0.033 (sol) & 1.615 & 2.59 & 4.18 & 0.042 & 0.37 & 2.78 \\
\hline 0.040 (sol) & 1.820 & 2.51 & 4.57 & 0.044 & 0.35 & 2.70 \\
\hline 0.060 (sol) & 2.130 & 2.15 & 4.58 & 0.050 & 0.38 & 2.66 \\
\hline 0.070 (sol) & 2.180 & 1.93 & 4.21 & 0.056 & 0.40 & 2.54 \\
\hline 0.500 (gel) & & 1.23 & & 0.112 & 0.30 & 2.50 \\
\hline
\end{tabular}

${ }^{\text {a } T h i s ~ v a l u e ~ o f ~} D_{M}$ was obtained from the charge vs volume plots as indicated in Ref. [16].

$$
Q \propto M \propto r^{D_{M}},
$$

where $Q$ and $M$ are the Ag electrodeposition charge and mass, respectively. For $r<r_{c}, \log _{10} Q$ vs $\log _{10} r$ linear plots result [Figs. 3(a) and 3(b)], their slopes decreasing from $D_{M}=3$ to $D_{M} \cong 2.5$ as agarose concentration is increased (Table I). For $r>r_{c}$ those plots show a slight positive deviation from linearity which is enhanced in the gel [Fig. 3(b)], indicating a change in the object density.

The density change in the gel can be better seen through the proportionality

$$
\rho \propto r^{D_{M}-3},
$$

where $\rho$, the object apparent density (Ag and void domains), results from the $w_{\mathrm{Ag}} Q / F V$ ratio, where $w_{\mathrm{Ag}}$ is the Ag atomic weight, $F$ is Faraday's constant, and $V$ is the object apparent volume, $V=(4 / 3) \pi r^{3}$. Then, $D_{M}$ can be obtained from the $\log _{10} \rho$ vs $\log _{10} r$ plot (Fig. 4). This plot shows a linear decrease of $\log _{10} \rho$, with $\log _{10} r$ reaching $r_{c}$, the minimum value $\rho \cong 1.58 \times 10^{-3} \mathrm{~g} \mathrm{~cm}^{-3}$, i.e., the density of $\mathrm{Ag}^{+}$ions in solution is approached. For $r<r_{c}$, Eq. (2) leads to $D_{M}=2.5 \pm 0.02$. Otherwise, for $r>0.4 \mathrm{~cm}$ (Fig. 4) the limiting value $\rho=2.8 \times 10^{-3}$ $\mathrm{g} \mathrm{cm}^{-3}$ is attained, i.e., $D_{M} \rightarrow 3$. Hence, a constantdensity, more compact object is formed. This is confirmed by inspecting the front view of an Ag electrodeposit grown in the gel (Fig. 5). The thickness of the outer low-density shell remains nearly constant as $r$ increases from $r_{c}$ upwards. For those objects grown in 0.07 $\mathrm{g}$ agarose $/ 100 \mathrm{ml}$ and $0.5 \mathrm{~g}$ agarose $/ 100 \mathrm{ml}$ and $r<r_{c}$, $\left\langle D_{M}\right\rangle=2.52 \pm 0.03$, a figure that is close to $D_{M}$ values reported for 3D Cu electrodeposits [2] and computer simulations of 3D DLA models [4].

The macroscopic behavior of $\mathrm{Ag}$ electrodeposits formed for $r<r_{c}$ in sols fits a Stokes-like relationship, as the product $v_{r} \eta^{\prime}$ remains practically constant (4. $4 \pm 0.3 \times 10^{-4} \mathrm{P} \mathrm{cm} \mathrm{s}^{-1}$ ) within the range of $\eta^{\prime}$ covered in this work (Table I). The value of $\delta$, the thickness of the diffusion layer, can be estimated from the quotient $D_{i} / v_{r}=\delta$, where $D_{i}$ is the diffusion coefficient of $\mathrm{Ag}^{+}$ ions in solution. The value of $D_{i}$ becomes practically $\eta^{\prime}$ independent; it results in $D_{i}=1.1 \times 10^{-5} \mathrm{~cm}^{2} \mathrm{~s}^{-1}$ for all media. The value of $\delta$ is always smaller than $r_{c}$ and it increases with agarose concentration. In addition, for constant $Q$, as $\eta^{\prime}$ is increased, $\rho, v_{r}$, and $D_{M}$ decrease, and the object size increases (Table I). Mass transport rate equations [19], for a constant density gradient at a plane surface, yield values of $\delta$ considerably smaller than in the gel. This difference reflects the influence of free convection on the Ag growth pattern. The significance of the comparative scale of $\delta$ and the aggregate has been examined for 2D growth [8-10]. The DLA morphology is associated with $r<\delta$. This effect is clearly shown in Table I as the increase of $\eta^{\prime}$ leads to an increase of $\delta$, and to a $D_{M}$ value consistent with DLA morphology. The transition from a DLA to a dense aggregate resembles that described from 2D electrodeposits grown far from equilibri-

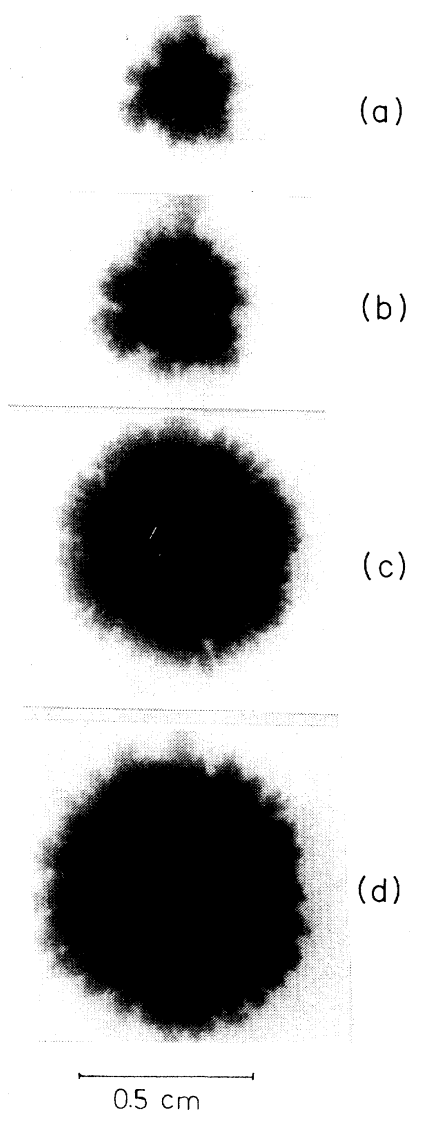

FIG. 5. Photographs of an $\mathrm{Ag}$ electrodeposit at different stages of growth. $E_{d}=-0.200 \mathrm{~V}, 0.50 M \quad \mathrm{Na}_{2} \mathrm{SO}_{4}=0.010 M$ $\mathrm{H}_{2} \mathrm{SO}_{4}+0.005 \mathrm{M} \quad \mathrm{Ag}_{2} \mathrm{SO}_{4}+0.5 \mathrm{~g}$ agarose $/ 100 \mathrm{ml}, 25^{\circ} \mathrm{C}$. (a) $Q=0.040 C$; (b) $Q=0.073 C$; (c) $Q=0.400 C$; (d) $Q=0.700 C$. The value of $r_{c}$ for this electrodeposit is $0.30 \mathrm{~cm}$. 

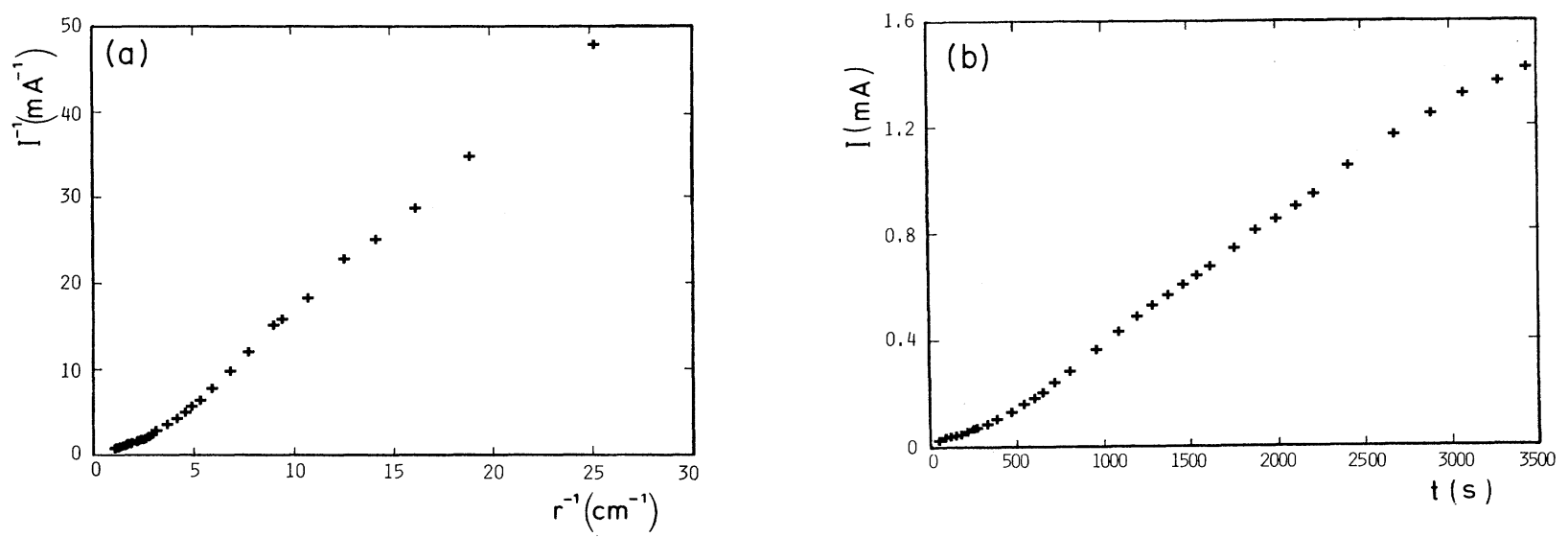

FIG. 6. (a) $I^{-1}$ vs $r^{-1}$ plot and (b) $I$ vs time plot for silver electrodeposits, $E_{d}=-0.200 \mathrm{~V}, 0.50 M \mathrm{Na}_{2} \mathrm{SO}_{4}+0.010 \mathrm{M}$ $\mathrm{H}_{2} \mathrm{SO}_{4}+0.005 \mathrm{M} \mathrm{Ag}_{2} \mathrm{SO}_{4}+0.07 \mathrm{~g}$ agarose $/ 100 \mathrm{ml}, 25^{\circ} \mathrm{C}$.

um $[20,21]$.

Ag electrodeposition in agarose-free solution produces density gradients at the solution adjacent to the electrode surface that induce a visible free convection effect at $t_{c}$. Then, for $r>r_{c}, \delta$ is no longer uniform, and the growth of an anisotropic and compact object $\left(D_{M}=3\right)$ is favored. In this case, individual branches may penetrate the diffusion layers, leaving pockets of undepleted solution between them.

On the other hand, isotropic Ag branched aggregates are grown in the gel irrespective of $r$, and in this case, an increase in compactness is observed. This effect may be due to penetration of branch tips into the diffusion field. Then, as $\delta$ increases, the diffusion layer forms a smooth envelope around the aggregate. Then, under damped free convection the growth of the object appears to be determined by the advance of the growing object front in the solution under a Laplacian field. Accordingly, a particular deposit morphology is related to a certain diffusion layer profile.

The cell can be modeled as two concentric spheres with $r$ and $r_{0}$ the inner and outer spheres radii, respectively. Then, the primary current distribution is given by

$$
I=4 \pi \kappa V_{a c} /\left[(1 / r)-\left(1 / r_{0}\right)\right],
$$

where $\kappa$ is the solution-specific conductance and $V_{a c}$ is the anode-cathode potential difference corrected for the overvoltage terms. Data given in Fig. 6(a) show that Eq. (3) is fulfilled when $Q<0.4 \mathrm{C}$. It should be noted that $I \propto r$ results for $r_{0} \gg r$. Then, the linear portion in the $r$ vs $t$ plot (Fig. 2) should correspond to a linear $I$-vs- $t$ relationship. This prediction of the Ohmic model is, in principle, fulfilled by experimental data [Fig. 6(b)].

This work was supported by the Gobierno de Canarias, Spain, under Contract No. 46/01.06.88 and the Consejo Nacional de Investigaciones Científicas y Técnicas (CONICET), Argentina.
[1] M. Matsushita, in The Fractal Approach to the Heterogeneous Chemistry, edited by D. Avnir (Wiley, New York, 1989), p. 161 and references therein.

[2] R. M. Brady and R. C. Ball, Nature 309, 225 (1984).

[3] M. Matsushita, M. Sano, Y. Hayakawa, H. Honjo, and Y. Sawada, Phys. Rev. Lett. 53, 286 (1984).

[4] P. Meakin, in The Fractal Approach to the Heterogeneous Chemistry (Ref. [1]), p. 131.

[5] B. Grier, E. Ben-Jacob, R. Clarke, and L. M. Sander, Phys. Rev. Lett. 56, 1264 (1986).

[6] T. A. Witten and L. M. Sander, Phys. Rev. Lett. 47, 1400 (1981).

[7] B. B. Mandelbrot, in The Fractal Geometry of Nature, (Freeman, New York, 1982).

[8] D. P. Barkey and P. D. LaPorte, J. Electrochem. Soc. 137, 1655 (1990).

[9] D. Barkey, J. Electrochem. Soc. 138, 2912 (1991).

[10] D. Barkey, P. Garik, E. Ben-Jacob, B. Miller, and B. Orr, J. Electrochem. Soc. 139, 1044 (1992).

[11] G. Daccord and R. Lenormand, Nature 325, 41 (1987).

[12] E. Chassaing, B. Sapoval, G. Daccord, and R. Lenormand, J. Electroanal. Chem. 279, 67 (1990).
[13] J. P. Kallungal and A. J. Barduhn, AIChE J. 23, 294 (1977).

[14] J. S. Huang and A. J. Barduhn, AIChE J. 31, 747 (1985).

[15] S. H. Tirmizi and W. N. Gill, J. Cryst. Growth 85, 488 (1987).

[16] A. Hernández-Creus, A. E. Bolzan, P. Carro, S. González, R. C. Salvarezza, S. L. Marchiano, and A. J. Arvia, J. Electroanal. Chem. 336, 85 (1992).

[17] A. Hernández-Creus, P. Carro, S. González, R. C. Salvarezza, and A. J. Arvia, J. Electrochem. Soc. 139, 1064 (1992).

[18] G. O. Aspinall, Polysaccharides (Pergamon, Oxford, 1970), p. 149.

[19] B. Levich, Physiochemical Hydrodynamics (Prentice-Hall, Englewood Cliffs, NJ, 1967).

[20] E. Ben-Jacob, P. Garik, T. Muller, and D. Grier, Phys. Rev. A 38, 1370 (1988); E. Ben-Jacob and P. Garik, Nature 343, 523 (1990).

[21] P. Garik, D. Barkey, E. Ben-Jacob, E. Botchner, N. Broxholm, B. Miller, B. Orr, and R. Zamir, Phys. Rev. Lett. 62, 2703 (1989). 
(a)

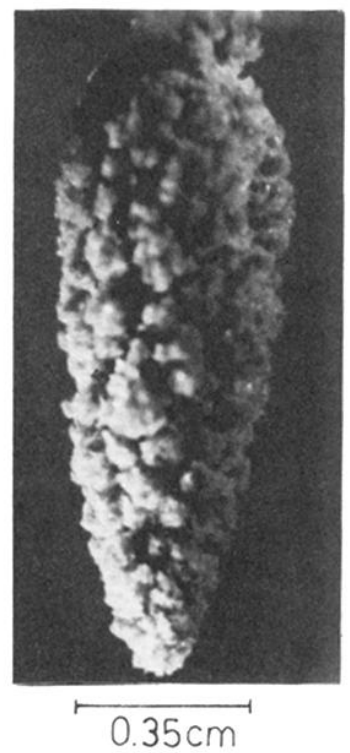

(c)

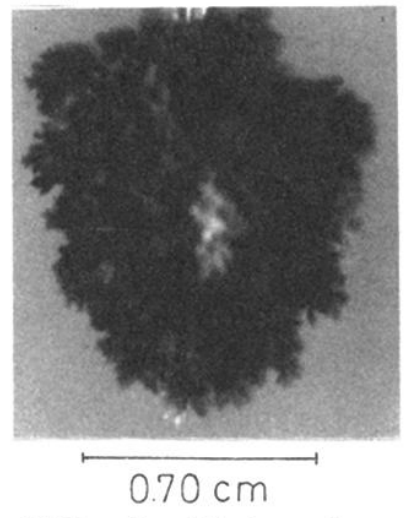

(b)

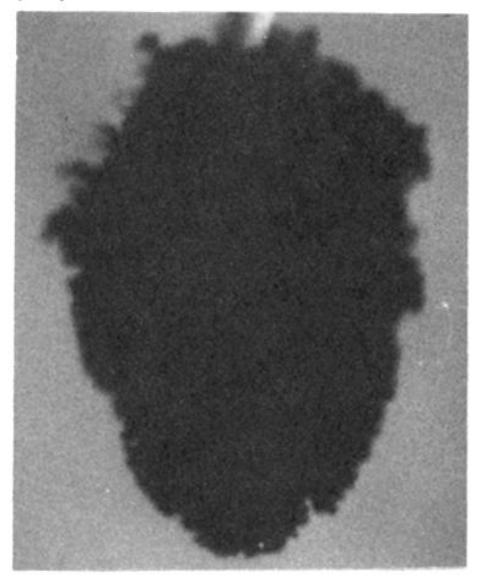

$0.50 \mathrm{~cm}$

(d)

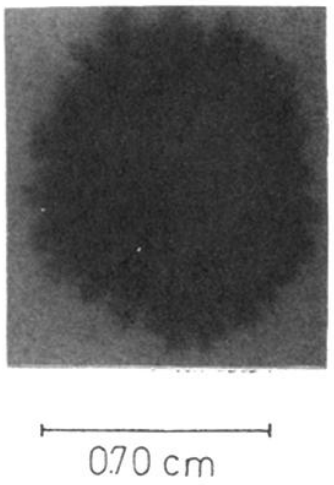

FIG. 2. Photographs of silver dendritic deposits. $E_{d}=-0.200 \mathrm{~V}, \quad 0.50 M \quad \mathrm{Na}_{2} \mathrm{SO}_{4}+0.010 M \quad \mathrm{H}_{2} \mathrm{SO}_{4}+0.005 M$ $\mathrm{Ag}_{2} \mathrm{SO}_{4}+x \mathrm{~g}$ agarose $/ 100 \mathrm{ml}, Q=1 C, 25^{\circ} \mathrm{C}$. (a) $x=0$; (b) $x=0.033$; (c) $x=0.070$; (d) $x=0.5$. 


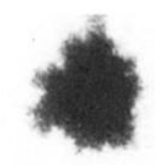

(a)

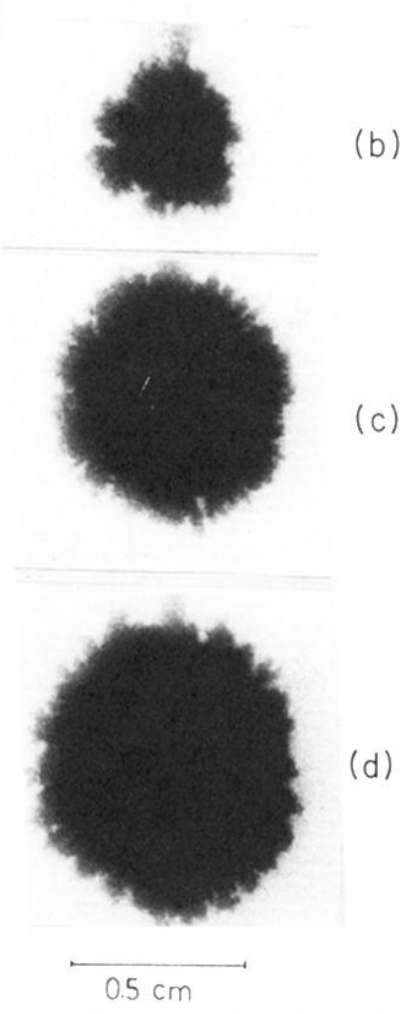

FIG. 5. Photographs of an $\mathrm{Ag}$ electrodeposit at different stages of growth. $E_{d}=-0.200 \mathrm{~V}, 0.50 M \mathrm{Na}_{2} \mathrm{SO}_{4}=0.010 \mathrm{M}$ $\mathrm{H}_{2} \mathrm{SO}_{4}+0.005 \mathrm{M} \mathrm{Ag} \mathrm{SO}_{4}+0.5 \mathrm{~g}$ agarose $/ 100 \mathrm{ml}, 25^{\circ} \mathrm{C}$. (a) $Q=0.040 C$; (b) $Q=0.073 C$; (c) $Q=0.400 C$; (d) $Q=0.700 C$. The value of $r_{c}$ for this electrodeposit is $0.30 \mathrm{~cm}$. 